\title{
Effects of the aeration on the fluid dynamic behaviour of a multi-zone activated sludge system
}

\author{
F. Sánchez ${ }^{1}$, A. S. Kaiser ${ }^{1}$, A. Viedma ${ }^{1}$ \& A. Gómez ${ }^{2}$ \\ ${ }^{1}$ Department of Thermal and Fluids Engineering, \\ Polytechnic University of Cartagena, Spain \\ ${ }^{2}$ Entidad de Saneamiento y Depuración de Aguas Residuales de la \\ Región de Murcia, Consejería de Agricultura y Agua, Spain
}

\begin{abstract}
Conventional wastewater treatment plants (WWTP) are necessary to modify the wastewater properties in order to make it acceptable for a safe discharge into the environment or a certain reuse purpose. Biological oxidation is the most important of the processes involved in conventional WWTP. Organic substances dissolved in the water are removed by means of bacteria presented in the biological reactor. Air is necessary to enable the reduction of the organic content of the water by the bacteria. Bubbles of air are introduced into the reactor through air diffusers. Air diffusers can account up to 70\% of WWTP total energy consumption.

So a deep understanding of the dynamic behaviour of the flow is necessary for optimizing the process and saving energy.

A numerical analysis of the effects of the aeration in the fluid dynamics behaviour of a real multi-zone activated sludge reactor is carried out. The purpose is to identify and analyse the changes originated in the velocity field by the aeration.

A numerical modelling of the activated sludge system located in San Pedro del Pinatar (Murcia, Spain) is developed throughout a general-purpose computational fluid dynamics (CFD) code. The multiphase flow is simulated with a EulerLagrange approach; modelling the bubbles as discrete phase. Two simulations, one with aeration and the other without it, are carried out. The numerical results show that the aeration has a notable effect in the performance of the reactor. Changes in velocity field, stagnant zones, residence time distribution or even free surface level originated by the aeration in the reactor are studied. In general, the
\end{abstract}


aeration reduced the amount of stagnant volume in the reactor. However, when the aeration is activated, some re-circulating zones are formed, reducing the residence time in the reactor.

Keywords: air bubbles-water interaction, activated sludge system, wastewater treatment plant, numerical modelling.

\section{Introduction}

Nowadays, people are more concerned about environment. The concept Sustainable Development is taken into account by governments and companies, which make a considerable economical effort to carry out the restrictive environmental laws. In this context, it is clear the necessity of treating city wastewater (sewage) before discharging it into the environment, or even treating it for other purposes such as irrigation. The sewage treatment is carried out in Waste Water Treatment Plants (WWTPs). Conventional WWTPs includes physical, chemical and biological processes to remove physical, chemical and biological contaminants. Biological oxidation is one of the most important processes involved in the treatment. Bacteria and other microorganisms (biological floc) remove organic substances dissolved in the water. The combination of wastewater and biological floc is called mixed liquor. Oxygen is necessary to enable the oxidation of the organic content of the sewage, air bubbles need to be added to the mix. Activated sludge plants are widely used to hold this biological process.

Activated sludge plants can account for up to $70 \%$ of total energy consumption of a WWTP, due mainly to the aeration process through the air diffusers [1]. So it is necessary a knowledge of the fluid dynamic phenomena which occur in the plant. This knowledge allows us to optimize the processes, saving energy and guaranteeing an efficient treatment. A numerical modeling of a real multi-zone activated sludge plant is carried out in this work. In addition to the air diffusers, the plant has mixers impellers to improve the mixed process. Computational Fluid Dynamics (CFD) has been widely used for simulating activated sludge systems. Bubble column have been numerically investigated either with Euler-Euler approach [2, 3] or with Euler-Lagrange approach [4]. Euler-Lagrange approach tends to have higher computational cost; however it allows us to study the trajectory of each individual bubble. Regarding the turbulence model, different turbulence model have been studied: Le Moullec et al. [5] analyzed two different turbulence models for an activated sludge system; he concluded that Reynolds Stress Model (RSM) gave more accurate residence time distribution (RTD) than the $k-\varepsilon$ model. Despite this, most of the works [6] use the well-known $k-\varepsilon$ model. In the work of Talvy et al. [7] a numerical modelling of a full scale oxidation ditch is carried out, simulating the mixer impellers as plane regions with a pressure jump between both sides of the planes.

Using the above mentioned bibliography, this work analyzes the effects of the air bubbles on the fluid dynamic behavior of a real multi zone activated sludge plant. The peculiarity of this plant is the complexity of the configuration: it has four zones, two of them with air diffusers on the ground and the other two without 
them. The zones are separated by partition walls, which have two holes in its inferior parts. It is interesting to study how the air bubbles modify the flow through the holes. The plant also has two mixer impellers in each zone, adding other fluid dynamic phenomenon to the aeration.

The aim of this work is to study the influence of the air bubbles in the fluid dynamic behavior of the plant. Two simulations are carried out, one with aeration and other without aeration. The hydraulic performance of the reactor is evaluated in terms of stagnant volume (percentage of liquid with low velocity). Other aspects like the influence of the aeration in the free surface level are mentioned. The results obtained provide useful information for the improvement of the efficiency of the plant, taking advantage of the effects produced by the bubbles.

\section{Installation description}

The numerical modeling developed in this work corresponds to the multi zone activated sludge reactor of the WWTP located in San Pedro del Pinatar (Murcia, Spain), see Figure 1. This reactor holds the biological oxidation of the treated sewage. It is divided into two parallel lanes, each one is formed by four zones. Each zone has two mixer impellers (FLYGT-S460) in two opposite corners. The impellers are installed forming a $20^{\circ}$ angle with the wall. The air diffusers (FLYGT membrane EDPM 9' ') are located uniformly on the floors of Zone 2 (616 diffusers) and Zone 4 (300 diffusers). The four zones are separated by three partition walls. The first partition wall has $5.53 \mathrm{~m}$ height, while the second and third ones have $5.05 \mathrm{~m}$ height. All the partition walls have two inferior holes of $0.4 \times 0.45 \mathrm{~m}$. So the water can pass to one zone to the next zone either, over the partition wall or through the inferior holes. The outlet weir is $5.43 \mathrm{~m}$ above the floor.

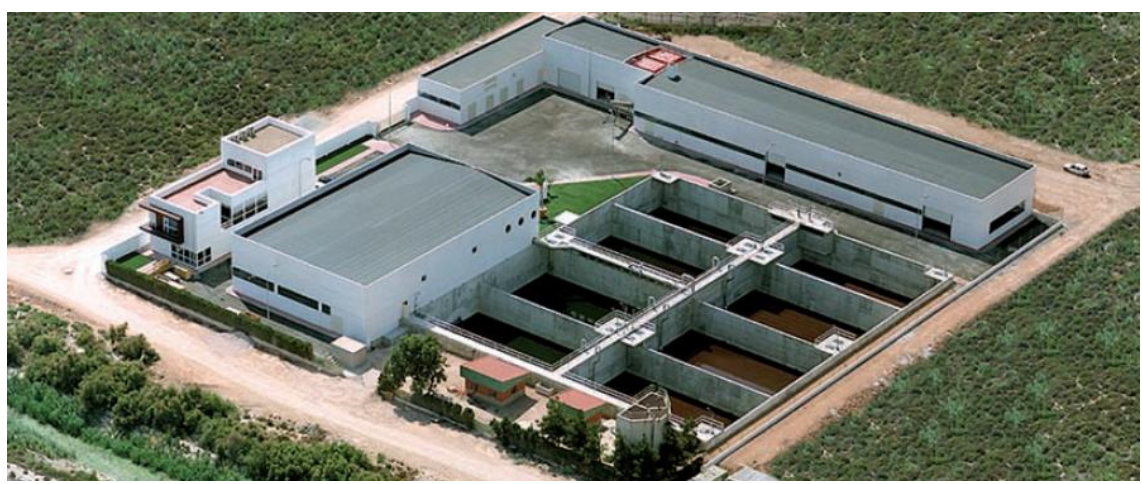

Figure 1: Aerial photography of the WWTP where the plant is located. 


\section{Numerical modelling}

\subsection{Governing equations}

An Eulerian-Lagrangian model is employed for simulating the two phases involved in the problem. The continuous phase (mixed liquor) is governed by mass and momentum principles, while the dispersed phase (air bubbles) is governed by a force balance from the Lagrangian point of view. Both phases are coupled through source terms.

\subsubsection{Mixed liquor flow governing equations}

The continuous phase is modelled by means of the simplified RANS steady equations:

$$
\begin{gathered}
\frac{\partial\left(\rho U_{j}\right)}{\partial j}=0 \\
\frac{\partial\left(\rho U_{i} U_{j}\right)}{\partial x_{j}}=\frac{\partial}{\partial x_{j}}\left[\mu\left(\frac{\partial U_{i}}{\partial x_{j}}+\frac{\partial U_{j}}{\partial x_{i}}\right)-\frac{2}{3} \mu\left(\frac{\partial U_{j}}{\partial x_{j}}\right) \delta_{i j}-\rho \overline{u_{\imath} u_{j}}\right]-\frac{\partial p}{\partial x_{i}}+\rho g_{i}
\end{gathered}
$$

where $U$ is the averaged velocity and $p$ the relative pressure, difference between the pressure and the ambient pressure. The turbulent stress $-\overline{u_{\imath} u_{J}}$ is provided from the turbulence closure model, assuming that

$$
-\overline{u_{\imath} u_{\jmath}}=2 v_{t} S_{i j}-\frac{2}{3} k \delta_{i j}
$$

being $v_{t}$ the turbulent kinematic viscosity; $S_{i j}$ is the mean strain tensor, $S_{i j}=\left[\left(\partial U_{i} / \partial x_{j}\right)+\left(\partial U_{j} / \partial x_{i}\right)\right] / 2, \delta_{i j}$ the Krönecker delta and $k$ the kinetic turbulent energy, given by $k=\sum_{j=1}^{j=3} \overline{u_{j}^{2}} / 2$. The well-know $k-\varepsilon$ turbulence model is employed to solve the closure problem. So, two additional equations for the transport of kinetic turbulent energy, $k$, and the dissipation rate of $k, \varepsilon$ are included in the modelling.

\subsubsection{Air bubbles governing equations}

Air bubbles are considered as discrete phase. Many bubbles are released uniformly from the floor of Zones 2 and 4 . The trajectory $r_{p}$ of each bubble is calculated by means of the integration of the force balance on the particle:

$$
\begin{gathered}
\frac{d r_{p}}{d t}=U_{p}, \\
\frac{d U_{p}}{d t}=F_{D}\left(U-U_{p}\right)+\frac{g_{r}\left(\rho_{p}-\rho\right)}{\rho_{p}}+\frac{\rho}{\rho_{p}} U_{p} \frac{\partial U}{\partial r_{p}}, \\
F_{D}=\frac{18 \mu}{\rho_{p} D_{p}^{2}} \frac{C_{D} R e_{p}}{24}, \\
C_{D}=a_{1}+\frac{a_{2}}{R e_{p}}+\frac{a_{3}}{R e_{p}^{2}},
\end{gathered}
$$

with $R e_{p}=\left(\rho D_{p}\left|U-U_{p}\right|\right) / \mu$ the Reynolds number of the particle. The coefficients $a_{1}, a_{2}$ and $a_{3}$ are constants used for smooth particles over several 
ranges of $R e$ (Morsi and Alexander [8]); $F_{D}\left(U-U_{p}\right)$ is the drag force per unit particle mass, $g_{r}\left(\rho_{p}-\rho\right) / \rho_{p}$ is the gravity force per unit particle mass, $\left(\rho / \rho_{p}\right) U_{p}\left(\partial U / \partial r_{p}\right)$ is the force due to the pressure gradient in the fluid.

The dispersion of particles due to turbulence in the continuous phase is simulated using a stochastic tracking model. In the RANS steady equations the velocity of the fluid is composed by a mean velocity $U_{j}$ and a random velocity fluctuation $u_{j}$. When the trajectory of a droplet is integrated (Equation (5)), $u_{j}$ is kept constant for a certain interval of time $t_{e}$, which depends on the characteristic time scale or lifetime of eddies. The lifetime of the eddies is written as $t_{e}=$ $-t_{l} \log (r)$. Where $t_{l}$ is the time scale of the Lagrangian flow, $t_{l}=C_{l} \frac{k}{\varepsilon}$, in the turbulence model $k-\varepsilon$, the value of $C_{l}$ is 0.15 . The term $r$ is a random number between 0 and 1 . The dispersion is simulated computing each trajectory for a sufficient number of particles (tries).

\subsubsection{Interaction between dispersed and continuous phase}

The effect of the air bubbles on the liquid is taken into account by means of the incorporation of source terms into the right terms of the equations of the liquid (Equations (1) and (2)). $F_{i}$ is the momentum communicated from the bubbles to the mixed liquor, this source term is added into the balance of kinetic momentum of the liquid (Equation (2)).

\subsection{Computational domain and meshing detail}

The domain of the problem includes the four zones described in the previous section, as well as the impellers and the inferior holes of the partition walls. The height of the domain has to mimic the free surface level, so it depends on the liquid flow rate and the aeration.

The mesh is mainly Cartesian and structured. The sizes of the elements vary from $0.02 \mathrm{~m}$ around the impellers to $0.14 \mathrm{~m}$ in the core of the domain. The mesh has 5.2 millions of elements.

\subsection{Solver settings and boundary conditions}

The numerical modelling are developed using a general-purpose CFD code, based on a finite volume procedure. The equations are discretized by a staggered-grid scheme. The coupling between mass and momentum equations are solved by the "SIMPLE" algorithm through pressure. The well-known "up-wind" second-order differencing scheme is employed for the convective terms of the equations. The case is considered converged when the normalized residual for mass, momentum and turbulent variables are lower than $10^{-5}$.

The sides and ground walls of the domain are treated as non-slip walls, while the conventional symmetry condition it used for the top surfaces, simulating the free surface level behaviour. The usual pressure-outlet boundary condition is used in the exit of the domain. The liquid goes into the domain with uniform velocity through the inlet surface. The mixer impellers are simulated by means of a pressure 
jump in a plane region, including the azimuthal velocity. The air bubbles are injected uniformly through the grounds of Zones 2 and 4.

\section{Numerical results}

Two simulations of the biological reactor have been carried out with the numerical modelling developed. Both of them have the same wastewater influent flow rate; in one simulation the air diffusers are working, in the other they are not working.

\subsection{Description of the flow}

The numerical results show a notable influence of the aeration on the velocity field in the reactor. Without aeration, a vortex is generated in each zone (Figure 2(a)). The vortex is generated because of the jets of the mixers impellers; the pair of mixer impellers of each zone are located in opposite corners of the zone, faced each other; the jets are parallel, but not aligned. This jets misalignment generates a big vortex in the center of the zone. However, when the air diffusers of Zones 2 and 4 start to work, the columns of air bubbles generate an upward force in the liquid. This upward force adds a vertical component to the liquid velocity, splitting the vortex which there was without aeration in two or three smaller and weaker vortex (Figure 2(b)). This phenomenon is stronger in Zone 2 than in Zone 4, because Zone 2 has more air diffusers and therefore more air flow rate.

Another notable effect of the aeration on the liquid is the circulation through the inferior holes of the partition walls. Without aeration, there is hardly liquid circulation through the inferior holes of the second and third partition walls. However, when the air diffusers are activated, the upward force of the bubbles in the liquid generates a depression in the lower part of the Zones 2 and 4 (Figure 3). This depression favours the circulation of the mixed liquor from Zone 3 to Zone 4 and from Zone 3 to Zone 2, producing a recirculation. As explained in the installation description, the first partition wall is higher than the other two, causing two different free surface levels: one for the Zone 1 and another for the other three zones. Without aeration, the free surface level in Zone 1 is a little higher than the first partition wall: a little fraction of the liquid flow rate passes over the wall, while the rest is drawn off by the inferior holes. Nevertheless, when the air diffusers start to work, because of the depression generated on the bottom of Zone 2, all the liquid is drawn off by the inferior holes, decreasing $8 \mathrm{~cm}$ the free surface level of Zone 1. The free surface level in the other three zones is not affected by the aeration.

Air bubbles also have influence on the residence time of the waste water in the reactor. The average residence time is useful for calculating which fraction of the reactor volume is active volume (not recirculating volume). Without aeration, the average residence time of the liquid in the reactor is 361 minutes, whereas when the air diffusers are working the average residence time in the biological reactor is 321 minutes. These residence times mean that without aeration the reactor active volume is $82.5 \%$, with aeration the active volume decreases until $73.3 \%$. 


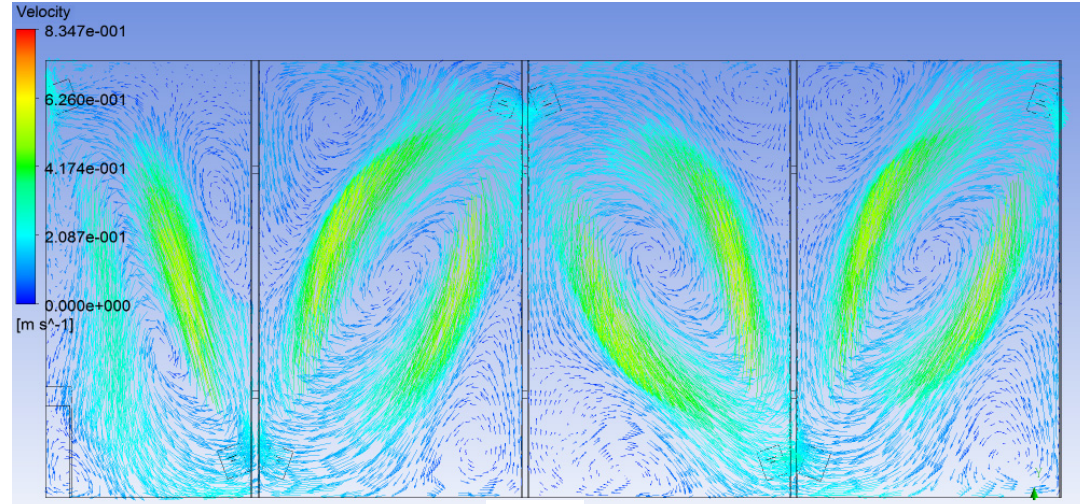

(a)

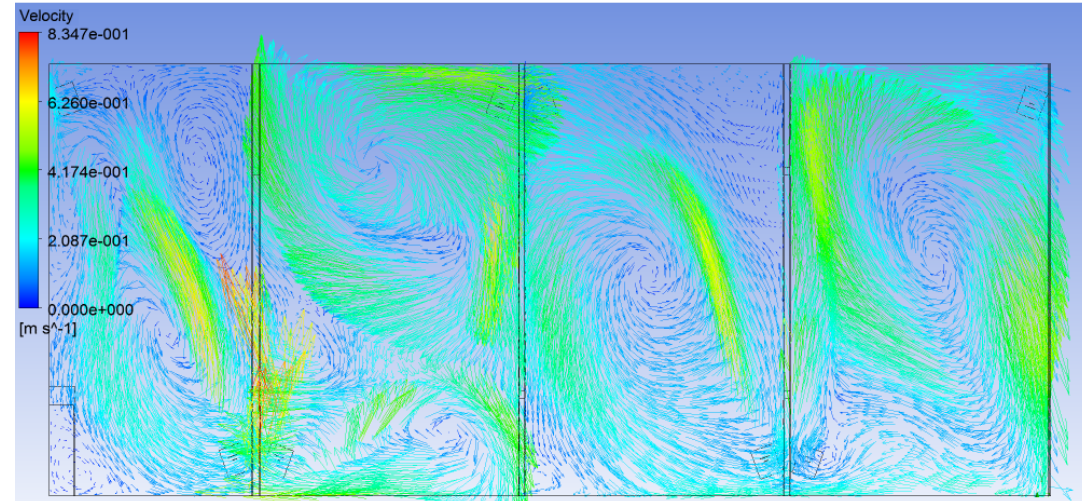

(b)

Figure 2: Velocity vectors in the horizontal plane $\mathrm{z}=2.5 \mathrm{~m}$ (a) for the simulation without aeration; (b) for the simulation with aeration. The aeration divides the vortex of Zones 2 and 4 into smaller vortexes.

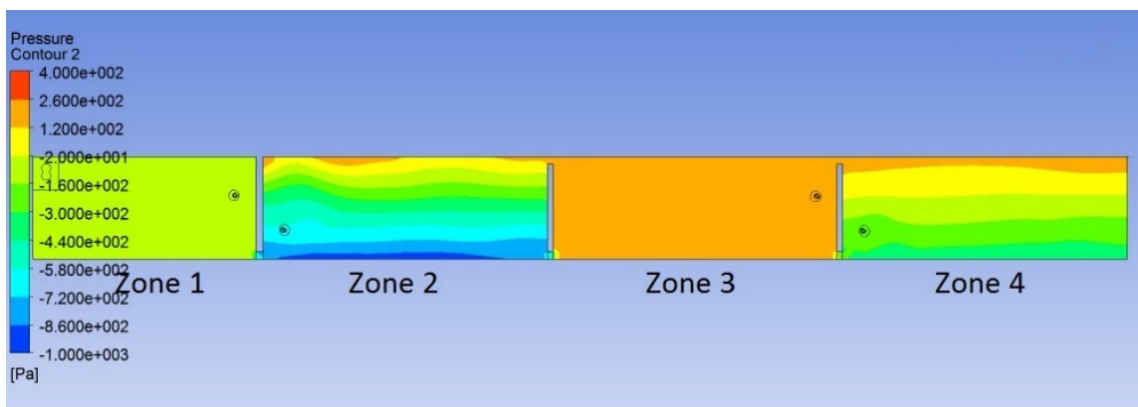

Figure 3: Hydraulic head in the vertical plane $y=6 \mathrm{~m}$ for the simulation with aeration. It is observed the depressions produced by the air bubbles. 


\subsection{Effect of the aeration on the reactor performance}

A quantitative analysis of the velocity field is carried out. Figure 4 shows the histogram with the percentage of volume of liquid in each velocity range. The aeration has clear effects on the distribution of velocities. Without aeration, most of the liquid has velocity lower than $0.2 \mathrm{~m} / \mathrm{s}$. However, when the air diffusers start to work, the percentage of liquid with low velocity reduces, and the averaged velocity in the reactor increases. This increase of motion is due to the upward force of the air bubbles on the liquid, which generates the movement of zones that did not have velocity without aeration.

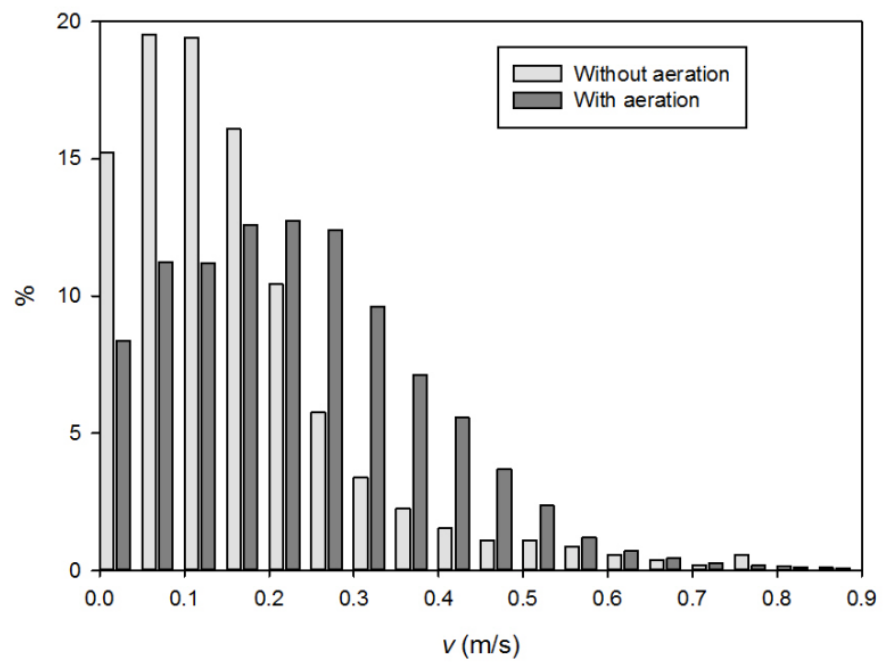

Figure 4: Comparison of the velocity histograms obtained without and with aeration.

The zones with velocity lower than $0.1 \mathrm{~m} / \mathrm{s}$ are considered as stagnant zones. The analysis of stagnant zones is very important in WWTP, because the sedimentation of the suspended solid is only allowed to take place in stagnant zones. Table 1 shows the stagnant volume in each zone of the biological reactor in the two cases simulated. Without aeration, more than $37 \%$ of the liquid is stagnant volume. When the air diffusers are working, the stagnant volume reduces until 19\%. Studying the stagnant volume in each zone, it is observed that the zones with air diffusers (zones 2 and 4) reduce their stagnant volume more than the other two zones. This is because the air bubbles induce the movement of the mixed liquor of the zones, and by means of the flow circulation through the inferior holes of the partition wall also reduce the stagnant volume in Zone 3. Figure 5 shows the stagnant volume in the two simulations. Without aeration (Figure 5(a)) the zones with velocity lower than $0.1 \mathrm{~m} / \mathrm{s}$ locate in the centre of the vortex and the corners and walls of the zones. Nevertheless, with aeration (Figure 5(b)) the stagnant volume in Zones 2 and 4 are greatly reduced. 
Table 1: Stagnant zone percentage $(\mathrm{v}<0.1 \mathrm{~m} / \mathrm{s})$ in each zone and in the whole reactor.

\begin{tabular}{|c|c|c|c|c|c|}
\cline { 2 - 6 } \multicolumn{1}{c|}{} & Zone 1 & Zone 2 & Zone 3 & Zone 4 & Total \\
\hline Without air & $46.13 \%$ & $38.93 \%$ & $35.88 \%$ & $32.65 \%$ & $37.45 \%$ \\
\hline With air & $48.96 \%$ & $3.58 \%$ & $22.97 \%$ & $9.50 \%$ & $19.61 \%$ \\
\hline
\end{tabular}


Figure 5: Fluid with velocity lower than $0.1 \mathrm{~m} / \mathrm{s}$ (a) in the simulation without aeration; (b) in simulation with aeration. The stagnant volume decreases when the aeration is activated.

\subsection{Bubble distribution}

According to [9], the Euler-Lagrange numerical approach is only possible if the particle volume fraction is less than $10 \%$. Figure 6 shows the bubbles volume 
fraction in a vertical plane of the domain. As expected, there are bubbles only in the zones with diffusers; being higher bubble concentration in the Zone 2 than in Zone 4 (Zone 2 has 696 air diffusers, while Zone 4 has 300 air diffusers). The particle volume fraction hardly reaches $4 \%$, far less than the limit $10 \%$. One of the advantages of using an Euler-Lagrange model is that it allows us to study the trajectory of each particle. As explained before, air diffusers represent a large fraction of the total energy consumptions in WWTPs. For this reason is important to optimize the aeration, injecting the right mass flow rate of air, keeping the air bubbles in the reactor the necessary time to enable the oxidation, but using as little energy as possible. Reducing the size of the air bubbles injected is possible to increase the residence time of the bubbles in the reactor. However, with small bubbles, the air flow rate per diffuser is lower, thus more diffusers would be needed, increasing the energy consumption. In the biological reactor analysed in this work, where the bubbles diameter is $1.016 \mathrm{~mm}$, the mean residence time of the bubbles in the reactor is $64 \mathrm{~s}$ for the bubbles injected from Zone 2 and $68 \mathrm{~s}$ for the bubbles injected from Zone 4.

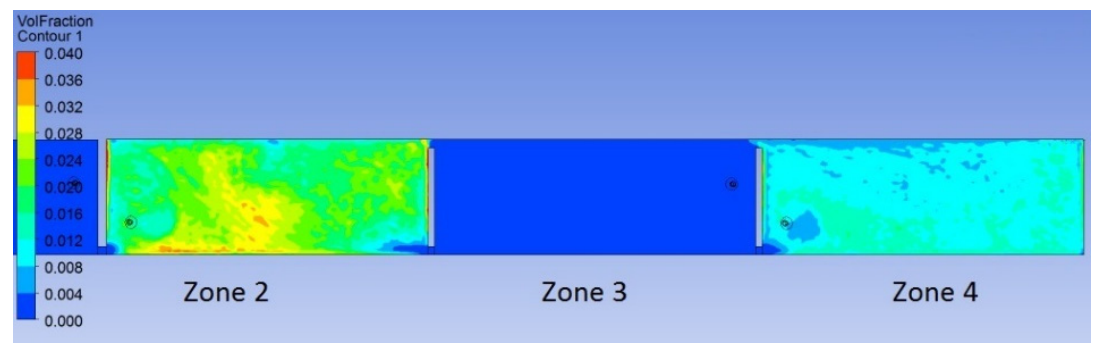

Figure 6: Volume fraction of air in the vertical plane $y=6 \mathrm{~m}$ of the simulation with aeration.

\section{Conclusions}

A numerical modelling of the multi-zone activated sludge system of the wastewater treatment plant (WWTP) located in San Pedro del Pinatar (Murcia, Spain) is carried out. Relevant parameters as velocity field, stagnant zones or residence time are studied under two different working regimes, without aeration and with the air diffusers working. The following conclusions remark can be made:

- Without aeration, a consistent vortex is generated in each one of the four zones of the reactor. With aeration, the vortexes of the zones with air diffusers (Zones 2 and 4) split into smaller and weaker vortexes, due to the upward force of the air bubbles on the liquid.

- The aeration generates the movement of the liquid through the inferior holes of the second and the third partition walls, this is because of the depression produced by the air bubbles in the zones of the air diffusers. 
- The depression generated by the air bubbles on the ground of Zone 2 produces the decrease of the free surface level of Zone 1. Free surface level of Zone 1 is $5.533 \mathrm{~m}$ without aeration and $5.451 \mathrm{~m}$ with aeration.

- The air bubbles have notable influence in the residence time of the liquid in the reactor, decreasing the active volume from $82.5 \%$ (without aeration) to $72.3 \%$ (with aeration).

- The aeration generates a decrease in the amount of stagnant volume in the reactor. The amount of liquid with velocity lower than $0.1 \mathrm{~m} / \mathrm{s}$ without aeration is $37.5 \%$, however, with the air diffusers activated, only $19.6 \%$ of the liquid of the reactor has velocity lower than $0.1 \mathrm{~m} / \mathrm{s}$.

\section{Acknowledgement}

The researcher team of the present work acknowledges the contribution of the company ESAMUR, owner of the biological reactor studied, which provided the experimental data necessary for the development of the numerical model presented.

\section{References}

[1] Fayolle, Y., Cockx, A., Gillota, S., Roustan, M. and Héduita, A. (2007). Oxygen transfer prediction in aeration tanks using CFD. Chemical Engineering Science 62, pp. 7163-7171.

[2] Cockx, A., Liné, A., Roustan, M., Do-Quang, Z. and Lazarova, V. (1997). Numerical simulation and physical modeling of the hydrodynamics in an airlift internal loop reactor. Chemical Engineering Science 52, pp. 3787-3793.

[3] Gresch, M., Armbruster, M., Braun, D. and Gujer, W. (2011). Improving the hydraulic efficiency of water process tanks using CFD models. Water Research 45, pp. 810-818.

[4] Gong, X., Takagi, S., Huang, H. and Matsumoto, Y. (2007). A numerical study of mass transfer of ozone dissolution in bubble plumes with an EulerLagrange method. Chemical Engineering Science 62, pp. 1081-1093.

[5] Le Moullec, Y., Potier, O., Gentric, C. and Leclerc, P. J. (2008). Flow field and residence time distribution of a cross-flow gas-liquid wastewater treatment reactor using CFD. Chemical Engineering Science 63, pp. 24362449.

[6] Yang, Y., Yang, J., Zuo, J., Li, Y., He, S., Yang, X. and Zhang, K. (2011). Study on two operating conditions of a full-scale oxidation ditch for optimization of energy consumption and effluent quality by using CFD. Water Research 45, pp. 3439-3452.

[7] Talvy, S., Debaste, F., Martinelli, L., Chauveheid, E. and Haut, B. (2011). Development of a tool, using CFD, for assessment of the disinfection process by ozonation in industrial scale drinking water treatment plants. Chemical Engineering Science 66, pp. 3185-1394.

[8] Morsi, S. A. and Alexander, A. J. (1972). An investigation of particle trajectory in two-phase flow systems. J. Fluids Mechanics 55, pp. 193-208.

[9] ANSYS INC. (2012). Ansys Fluent 14.0 Theory Guide. 